\title{
Acurácia da cintilografia com 99mTc-ubiquicidina (29-41) no diagnóstico diferencial de infecções musculoesqueléticas e processos inflamatórios estéreis
}

\author{
Accuracy of 99mTc-ubiquicidin (29-41) scintigraphy on the differential diagnosis \\ of musculoskeletal infectious diseases and sterile inflammations
}

\author{
Felipe Rocha Silva ${ }^{1}$, Larissa Araújo Dutra de Carvalho ${ }^{1}$, Bruna Evellyn de Lima Alves ${ }^{1}$, \\ Ana Raquel Souza de Azevedo ${ }^{1}$, Cejana de Mello Campos' \\ Osvaldo Sampaio Netto ${ }^{3}$, Roberto José Bittencourt ${ }^{4}$
}

Silva FR, Carvalho LAD, Alves BEL, Azevedo ARS, Campos CM, Netto OS, Bittencourt RJ. Acurácia da cintilografia com 99mTcubiquicidina (29-41) no diagnóstico diferencial de infecções musculoesqueléticas e processos inflamatórios estéreis / Accuracy of 99mTc-ubiquicidin (29-41) scintigraphy on the differential diagnosis of musculoskeletal infectious diseases and sterile inflammations. Rev Med (São Paulo). 2017 out.-dez.;96(4):225-31.

RESUMO: O diagnóstico diferencial entre infecção e processo inflamatório não infeccioso é crítico na avaliação de um paciente e sua análise por exames de imagem sensíveis e específicos se faz necessária. O objetivo desta revisão sistemática é determinar a acurácia da cintilografia com ${ }^{99 m}$ Tc-ubiquicidina (29-41) no diagnóstico diferencial de condições infecciosas e inflamações estéreis. A busca dos artigos foi realizada nas bases de dados de acesso livre Scientific Electronic Library Online (SciELO), PubMed, LILACS, Biblioteca Virtual em Saúde (BVS), Cochrane Library, Banco de Teses e Dissertações da CAPES e Health System Evidences com os descritores "ubiquicidin", "ubiquicidina" ou "UBI" presentes no título ou resumo de artigos publicados entre janeiro de 2000 até janeiro de 2017. A análise dos artigos incluídos sugere que a cintilografia com ${ }^{99 \mathrm{~m}} \mathrm{Tc}-$ ubiquicidina (29-41) é um método de elevada acurácia para o diagnóstico diferencial entre infecções musculoesqueléticas e inflamações assépticas.

Descritores: Cintilografia; Medronato de tecnécio Tc99m; Doenças musculares/diagnóstico por imagem; Confiabilidade dos dados; Diagnóstico diferencial.
ABSTRACT: The differential diagnosis between infection and a sterile inflammatory process is critical and requires imaging techniques that are sensitive and specific. The objective of this systematic review is to determine the accuracy of the ${ }^{99 \mathrm{~m}} \mathrm{Tc}$ ubiquicidin (29-41) scintigraphy on the differential diagnosis of musculoskeletal infectious diseases and sterile inflammations. The papers were searched on the free access databases Scientific Electronic Library Online (ScieLO), PubMed, LILACS, Biblioteca Virtual em Saúde (BVS), Cochrane Library, Banco de Teses e Dissertações da CAPES e Health System Evidences with the descriptors "ubiquicidin", "ubiquicidina" or "UBI" on the title or abstract of papers published from January 2000 to January 2017 . The analysis of the studies suggests that ${ }^{99 \mathrm{~m}} \mathrm{Tc}-$ ubiquicidin (29-41) scintigraphy is a method of high accuracy for the differential diagnosis of musculoskeletal infectious diseases and sterile inflammations.

Keywords: Radionuclide imaging; Technetium Tc99m medronate; Muscular diseases/diagnostic imaging; Data accurcy; Diagnosis, differential.

1. Universidade Católica de Brasília, Brasília, DF, BR. Acadêmico(a) do curso de Medicina. Email: rocha.fr7@gmail.com, larissa.dutrac@ gmail.com, brunaelalves@gmail.com, ana.rsazevedo@gmail.com.

2. Universidade Católica de Brasília, Brasília, DF, BR. Médica Nuclear, Adjunta da Clínica de Medicina Nuclear do Hospital das Forças Armadas, Docente do curso de Medicina da Universidade Católica de Brasília, Membro do Colégio Brasileiro de Radiologia. Email: cejana@gmail.com.

3. Universidade Católica de Brasília, Brasília, DF, BR. Médico Nuclear, Doutor, Chefe da Clínica de Medicina Nuclear do Hospital das Forças Armadas, Diretor do curso de Medicina da Universidade Católica de Brasília, Membro do Colégio Brasileiro de Radiologia. Email: osvsamp@gmail.com.

4. Universidade Católica de Brasília, Brasília, DF, BR. Médico, Doutor, Coordenador do Internato de Clínica Médica do curso de Medicina. Email: robertobitt@cardiol.br.

Endereço para correspondência: Felipe Rocha Silva. C3, lote 8, apt 401. Taguatinga, DF. CEP: 72010-030. 


\section{INTRODUÇÃO}

$\mathrm{O}$ diagnóstico diferencial entre um processo infeccioso e processo inflamatório não infeccioso é crítico na avaliação de um paciente com queixa musculoesquelética, já que essa informação pode alterar a conduta médica. Nem sempre a diferenciação clínica dessas condições é evidente, por isso pode ser necessário recorrer a exames de imagem para a elucidação diagnóstica.

Nesse contexto, técnicas específicas da medicina nuclear podem auxiliar o clínico. Um dos principais exames é a cintilografia, que pode ser realizada com leucócitos marcados, citrato de gálio-67 ou 99m-Tc-MDP (cintilografia óssea trifásica $)^{1,2}$. Esses radiofármacos possuem limitações no seu uso, como risco de contaminação durante a marcação dos leucócitos, longo tempo de preparo do material e dificuldades técnicas ${ }^{2,3}$. O radiofármaco ideal para a imagem de infecção deve satisfazer os seguintes critérios: alta captação pelo sítio infeccioso com alta especificidade, mínima captação pelos tecidos não-alvo, rápida deteç̧ão da infecção, rápida eliminação, baixa toxicidade, ausência de resposta imune e habilidade de diferenciar infecção de inflamação estéril ${ }^{1,4}$.

Diante da necessidade de novos radiofármacos que possam garantir imagens rápidas e precisas, com menos desvantagens em comparação àqueles utilizados atualmente, a cintilografia com ${ }^{99 \mathrm{~m}} \mathrm{Tc}$-ubiquicidina (2941) tem sido amplamente estudada. Esse método tem mostrado elevada acurácia em estudos que avaliam o diagnóstico diferencial entre infecção e inflamação estéril de tecidos ósseos, partes moles, pé diabético e próteses ${ }^{1-5}$.

A ubiquicidina é um polipeptídeo presente naturalmente em humanos ${ }^{6}$ que foi isolado inicialmente de macrófagos de camundongos ${ }^{7-8}$. Seu fragmento de aminoácidos 29-41 é carregado positivamente e se liga de maneira específica à superfície celular de bactérias e fungos, que são carregadas negativamente ${ }^{9-10}$. Esse peptídeo não se liga à superfície de bactérias mortas? O radiofármaco possui excreção renal ${ }^{10} \mathrm{com} 85 \%$ de eliminação em $24 \mathrm{~h}^{1}$, baixa absorção hepática e reduzida eliminação hepatobiliar ${ }^{10}$. Não apresenta efeitos cito ou genotóxicos ${ }^{11}$ e a marcação da ubiquidicina (29-41) com ${ }^{99 \mathrm{~m}} \mathrm{Tc}$ mostrou-se viável no Brasil ${ }^{12}$.

O objetivo desta revisão sistemática é determinar a acurácia da cintilografia com ${ }^{99 \mathrm{~m}} \mathrm{Tc}$-ubiquicidina (29-41) no diagnóstico diferencial entre infeccções e inflamações estéreis em tecidos musculoesqueléticos.

\section{METODOLOGIA}

Foi realizada uma revisão sistemática de estudos transversais de acurácia que investigaram a sensibilidade e especificidade da cintilografia com ${ }^{99 m} \mathrm{Tc}$-ubiquicidina (29-41) no diagnóstico diferencial de condições infecciosas e inflamatórias estéreis. As seguintes bases de dados de acesso livre foram pesquisadas: Scientific Electronic Library Online (SciELO), PubMed, LILACS e Biblioteca Virtual em Saúde (BVS), Cochrane Library, Banco de Teses e Dissertações da CAPES e Health System Evidences. As palavras chaves/descritores utilizadas para a busca foram "ubiquicidin", "ubiquicidina" ou "UBI" presentes no título ou resumo de artigos publicados entre janeiro de 2000 até janeiro de 2017. Os artigos foram analisados de maneira independente por dois autores. Foram considerados elegíveis para essa revisão todos os estudos clínicos em humanos publicados em português, espanhol ou inglês que relatam a sensibilidade e especificidade do método citado; relatos de caso e artigos de revisão foram excluídos. Dos onze estudos que respeitaram os critérios de inclusão, dez estão descritos neste trabalho, pois não foi possível acessar um dos artigos mesmo após tentativa de contato com os autores.

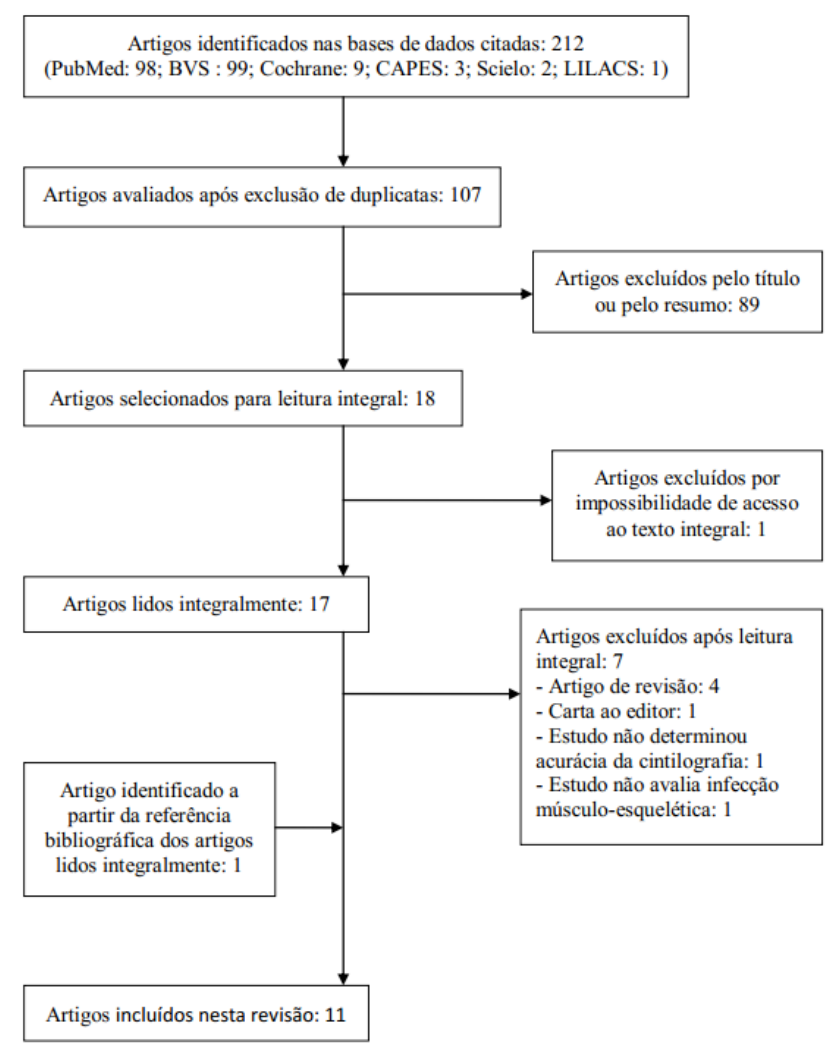

Figura 1. Metodologia 


\section{RESULTADOS}

Tabela 1: Resultados dos estudos analisados

\begin{tabular}{|c|c|c|c|c|c|c|}
\hline Autor, ano & Amostra & Sensibilidade & Especificidade & Acurácia & $\begin{array}{l}\text { Tempo de obtenção das } \\
\text { imagens (min) }\end{array}$ & Análise \\
\hline $\begin{array}{l}\text { Meléndez-Alafort, } \\
2004\end{array}$ & 6 & $100 \%$ & $100 \%$ & $100 \%$ & $1,30,120,240 \mathrm{~min}$ e $24 \mathrm{~h}$ & QL \\
\hline Akhtar, 2005 & 18 & $100 \%$ & $80 \%$ & $94 \%$ & $30^{\mathrm{a}}, 60,120$ & QL \\
\hline Gandomkar, 2009 & 7 & $100 \%$ & $100 \%$ & $100 \%$ & $30^{\mathrm{a}}, 60,120$ & QT \\
\hline $\begin{array}{l}\text { Fard-Esfahani, } \\
2010\end{array}$ & 15 & $40 \%$ & - & - & $60^{\mathrm{b}}, 120^{\mathrm{b}}$ & QL \\
\hline De Murphy, 2010 & 148 & $96 \%$ & $94 \%$ & $95 \%$ & 30,120 & QL \\
\hline Assadi, 2011 & 20 & $100 \%$ & $100 \%$ & $100 \%$ & $15^{\mathrm{a}}, 30,45,60,120$ e 240 & QT \\
\hline $\begin{array}{l}\text { Dillman-Arroyo, } \\
2011\end{array}$ & 27 & $100 \%$ & $88 \%$ & $96 \%$ & 120 & QL \\
\hline Aryana, 2012 & 34 & $100 \%$ & $100 \%$ & $100 \%$ & 30 & QT;QL \\
\hline Saeed, 2013 & 55 & $100 \%$ & $100 \%$ & $100 \%$ & $30^{\mathrm{a}}, 60$ & QL \\
\hline Beiki, 2013c & 9 & $100 \%$ & $100 \%$ & $100 \%$ & $30^{\mathrm{b}}, 60^{\mathrm{b}}, 120^{\mathrm{b}}$ & QT \\
\hline Esmailiejah, 2015 & 50 & $95 \%$ & $83 \%$ & $92 \%$ & $60^{\mathrm{a}}, 120$ & QT \\
\hline Total & 402 & & & & & \\
\hline
\end{tabular}

a) Melhor tempo para análise da imagem (quando essa informação foi analisada pelo autor)

b) $\mathrm{O}$ autor não encontrou diferença entre os tempos de obtenção da imagem

c) Os dados do autor apresentam uma inconsistência que será apresentada na discussão

$\mathrm{QL}=$ Qualitativa; QT = Quantitativa

Em um estudo de 2013, Saeed et al. ${ }^{13}$ selecionaram 55 indivíduos com suspeita clínica de osteomielite em pé diabético. Todos foram submetidos a uma cintilografia óssea trifásica com ${ }^{99 \mathrm{~m}} \mathrm{Tc}-\mathrm{MDP}$ e, após dois dias, a uma cintilografia com ${ }^{99 \mathrm{~m}} \mathrm{Tc}$-ubiquicidina (29-41). No protocolo do estudo, considerou-se o diagnóstico de osteomielite quando havia captação concordante de ${ }^{99 \mathrm{~m}} \mathrm{Tc}-\mathrm{MDP}$ e ${ }^{99 \mathrm{~m}} \mathrm{Tc}-$ ubiquicidina e de celulite quando a captação foi positiva para ${ }^{99 \mathrm{~m}}$ Tc-ubiquicidina e negativa para ${ }^{99 \mathrm{~m}} \mathrm{Tc}-\mathrm{MDP}$. A confirmação ou exclusão de osteomielite foi realizada a partir de avaliação histopatológica de biópsia óssea em 37 pacientes e por meio de critérios clínico-radiológicos após um acompanhamento mínimo de 3 meses nos demais 18 participantes. Neste estudo a ${ }^{99 \mathrm{~m}} \mathrm{Tc}$-ubiquicidina revelou sensibilidade, especificidade e acurácia de $100 \%$ para o diagnóstico de osteomielite. As imagens foram obtidas após 30 e 60 minutos da administração do fármaco. Os autores concluíram que o melhor momento para obtenção de imagens foi aos 30 minutos.

Assadi et al. ${ }^{14}$ avaliaram a acurácia da ${ }^{99 \mathrm{~m}} \mathrm{Tc}-$ ubiquicidina (29-41) em comparação com a acurácia da ${ }^{99 m}$ Tc-MDP e da ressonância magnética em 20 pacientes com suspeita de osteomielite em pé diabético. O padrãoouro para confirmação da osteomielite nesse estudo foi o consenso clínico baseado na apresentação da doença, exame histopatológico, cultura da ferida, exploração com haste metálica (probing), resposta à antibioticoterapia e exames laboratoriais. Os profissionais não tiveram acesso aos exames de imagem. Todos os 20 pacientes foram submetidos às cintilografias com ${ }^{99 \mathrm{~m}} \mathrm{Tc}$-ubiquicidina e ${ }^{99 m} \mathrm{mc}-\mathrm{MDP}$, mas apenas 12 foram submetidos à ressonância além dos outros 2 exames. Nesse estudo, determinou-se que a sensibilidade, especificidade e acurácia da ${ }^{99 \mathrm{~m}} \mathrm{Tc}$-ubiquicidina foram de $100 \%$ para o diagnóstico de osteomielite, enquanto os respectivos valores para a ${ }^{99 \mathrm{~m}} \mathrm{Tc}-\mathrm{MDP}$ foram de $100 \%$, 33\% e 90\% e da ressonância magnética foram de $67 \%, 100 \%$ e $75 \%$. As imagens com ${ }^{99 \mathrm{~m}} \mathrm{Tc}$-ubiquicidina foram obtidas aos 15 , 30, 45, 60, 120 e 240 minutos após a administração do radionuclídeo. Os autores determinaram que as imagens obtidas com 15 minutos possuíam a melhor relação órgão-alvo/fundo, o que tornou esse o melhor intervalo para avaliação diagnóstica do exame. Não houve relato de efeitos adversos até 14 dias após a realização do exame.

Fard-Esfahani et al. ${ }^{5}$ estudaram 15 pacientes portadores de pé diabético com suspeita de osteomielite ou celulite. Os pacientes foram submetidos a uma cintilografia óssea trifásica e a uma cintilografia com 
${ }^{99 m}$ Tc- ubiquicidina. Foram adquiridas imagens estáticas após 60 e 120 minutos da injeção do radiofármaco. Um escore visual foi utilizado para classificar o estudo como positivo ou negativo. Todos os exames foram avaliados por 3 médicos nucleares, sendo que o padrão-ouro para confirmação diagnóstica foi a cultura bacteriana. Nesse estudo, a sensibilidade da cintilografia com ${ }^{99 m} \mathrm{Tc}-$ ubiquicidina foi somente de $40 \%$ na identificação de infecção. Quando avaliado apenas o subgrupo portador de osteomielite, a técnica apresentou sensibilidade de $50 \%$. Nesse estudo não foi possível obter o valor de especificidade do exame, pois todas as culturas foram positivas.

Dillman-Arroyo et al. ${ }^{15}$ avaliaram a acurácia da ${ }^{99 m}$ Tc-ubiquicidina no diagnóstico de osteomielite vertebral em 27 pacientes. A confirmação ou exclusão de infecção foi feita por meio de análise histopatológica, cultura ou acompanhamento clínico por um tempo mínimo de 6 meses. Nesse estudo, a sensibilidade, especificidade e acurácia da ${ }^{99 m} \mathrm{Tc}$-ubiquicidina foram de 100\%, 88\% e $96 \%$. As imagens foram obtidas após 120 minutos da administração do radiofármaco.

Um estudo foi realizado por Gandomkar et al. ${ }^{3}$ com 7 pacientes com suspeita de infecção óssea ou de partes moles, antes que iniciassem qualquer uso de antibióticos. Estes receberam bolus de $\left[{ }^{99 \mathrm{~m}} \mathrm{Tc} /\right.$ Tricine/ HYNIC] ubiquicidina (29-41) e imagens dinâmicas da área alvo e de sua respectiva área contralateral foram obtidas 30, 60 e 120 minutos depois. Em 3 pacientes, foi observado imagem sugestiva de infecção de partes moles e confirmada após a cultura bacteriana; outros 2 pacientes com imagem positiva tiveram diagnóstico de infecção óssea confirmada por testes clínicos e radiografia. Para os 2 pacientes que obtiveram imagens negativas, foi sugerido processo inflamatório estéril de tecidos moles e confirmado por testes laboratoriais. Foi observado que imagens realizadas após 30 minutos da injeção do radiofármaco obtiveram a maior média de relação órgãoalvo/fundo. Os autores concluíram que esse método teve sensibilidade e especificidade de $100 \%$.

No estudo de Akhtar et al. ${ }^{2}$, selecionou-se um grupo de 18 pacientes com suspeita de osteomielite ou infecção bacteriana de partes moles que foram submetidos a cintilografia com ${ }^{99 m}$ Tc-ubiquicidina (29-41). Desses, 14 pacientes obtiveram imagens positivas, sendo que 10 eram de infecção de partes moles confirmada por cultura e três de infecção óssea confirmada por hemograma, radiografia e cintilografia óssea trifásica. A cultura foi negativa em 1 paciente com imagem positiva, que foi considerado como falso-positivo. A maior média na relação órgão-alvo/fundo foi após 30 minutos da injeção do radiofármaco. Esse estudo apresentou sensibilidade de $100 \%$, especificidade de $80 \%$ e acurácia de $94,4 \%$.

Meléndez-Alafort et al. ${ }^{1}$ avaliou em um grupo de 6 crianças a viabilidade do uso da ${ }^{99 \mathrm{~m}} \mathrm{Tc}$-ubiquicidia (29-41) na identificação de infecções em humanos. Foram obtidas imagens do corpo de crianças com suspeita de infecções ósseas após 1, 30, 120, 240 min e 24h da administração deste radiofármaco e comparadas com imagens obtidas com citrato de gálio-67, usado como controle, sendo que em dois casos a biópsia foi utilizada para confirmação do processo infeccioso. Este método apresentou uma sensibilidade e especificidade de $100 \%$ e nenhuma das crianças apresentou efeitos adversos.

O estudo de Murphy et al. ${ }^{4}$ teve como objetivo avaliar a sensibilidade, especificidade e acurácia da ${ }^{99 m}$ Tc-ubiquicidina (29-41) na detecção de vários tipos de infecções. Foram selecionados 148 pacientes com febre de origem desconhecida, osteomielite, pé diabético, infecção de prótese, artrite séptica e bacteremia para participar do ensaio clínico. A cultura bacteriana ou biópsia foram os critérios utilizados para confirmação do processo infeccioso. Concluiu-se que este radiofármaco tem um papel promissor na identificação dessas infecções, uma vez que apresentou sensibilidade de 96,3\%, especificidade de $94,1 \%$ e acurácia de $95,3 \%$.

Emailiejah et al. ${ }^{9}$ desenvolveram um estudo com 50 pacientes com suspeita de infecção musculoesquelética admitidos em um hospital ortopédico de referência no Irã entre 2011 e 2013. O objetivo era determinar a acurácia diagnóstica da cintilografia com ${ }^{99 \mathrm{~m}} \mathrm{Tc}$-ubiquidicina (2941), sendo a análise histológica considerada o padrãoouro do estudo. Determinou-se como suspeito de infecção musculoesquelética o quadro de dor em prótese de quadril ou joelho, dor em implante articular ou pseudoartrose. Foram adquiridas imagens dinâmicas e estáticas após 60 e 120 minutos da injeção do radiotraçador e feita análise quantitativa. Após a execução do exame, o procedimento cirúrgico foi realizado para confirmar a presença de infecção por meio de análise histopatológica. A técnica diagnóstica com o radiofármaco resultou em 95\% de sensibilidade, $83 \%$ de especificidade, valor preditivo positivo de $95 \%$, valor preditivo negativo de $83 \%$ e acurácia de $92 \%$. Em conclusão, segundo os autores, a cintilografia ${ }^{99 \mathrm{~m}} \mathrm{Tc}$-ubiquidicina é sensível e relativamente específica na localização de infecção óssea e de partes moles; o melhor tempo para a obtenção da imagem no estudo foi de 60 minutos após injetar o radiotraçador. $\mathrm{O}$ estudo concluiu ainda a necessidade de associar a história clínica e o exame físico ao possível agente etiológico para melhor acurácia do exame.

Beiki et al. ${ }^{10}$ promoveram um estudo com 9 pacientes com suspeita de infecção em implantes ortopédicos sem história prévia de antibioticoterapia. O que se pretendia era comparar a acurácia diagnóstica do padrão-ouro, a cultura do tecido periprotético, com a cintilografia ${ }^{99 m}$ Tc-ubiquidicina. Dos 9 pacientes, 5 tiveram infecção comprovada por cultura, todas por Staphylococcus aureus. A cintilografia interpretou 6 pacientes como infectados. As imagens foram adquiridas 
após 30, 60 e 120 minutos da injeção de ${ }^{99 m}$ Tc-ubiquidicina (29-41) e não foi visto diferença diagnóstica significativa em relação ao tempo. Os autores consideraram que o estudo obteve sensibilidade, especificidade, valor preditivo positivo, valor preditivo negativo e acurácia de $100 \%$. Beiki et al. ${ }^{10}$ concluíram que a cintilografia ${ }^{99 \mathrm{~m}} \mathrm{Tc}$-ubiquidicina tem alta acurácia para diagnóstico de infecção de próteses ortopédicas, mas afirmaram que a quantidade restrita de pacientes limitou o estudo.

Aryana et al. ${ }^{16}$ avaliaram a sensibilidade e a especificidade da cintilografia com 99mTc-ubiquicidina no diagnóstico diferencial entre infecção de prótese articular de quadril e soltura asséptica. Foram selecionados 34 pacientes de 20-79 anos de idade com queixa de dor em prótese de quadril nos quais não foi possível estabelecer clinicamente o diagnóstico. As imagens foram obtidas após 30 minutos da injeção do radiofármaco e a presença ou ausência de infecção foi confirmada a partir da combinação de achados cirúrgicos, microbiológicos e laboratorais. Nesse estudo foram realizados dois tipos de análise das imagens, ambos baseados na comparação entre a captação do radiofármaco na articulação suspeita e a captação na articulação contralateral normal. Na análise qualitativa, a comparação foi apenas visual; na análise semi-quantitativa um software determinou um quociente entre a captação do radiofármaco nas duas articulações e gerou a relação órgão-alvo/fundo. Os autores reportaram uma sensibilidade de $100 \%$ e especificidade de $92 \%$ para a análise qualitativa e uma sensibilidade e especificidade de $100 \%$ para a análise semi-quantitativa considerando uma relação órgão-alvo/fundo de 1,8.

\section{DISCUSSÃO}

Os exames padrão-ouro para o diagnóstico de osteomielite e infecção articular protética são a biópsia óssea com cultura ${ }^{17}$ e a cultura do tecido periprotético ${ }^{18}$, respectivamente. No entanto, por serem métodos invasivos, existe a necessidade de exames de imagem que apresentem elevada acurácia diagnóstica com resultados disponíveis rapidamente.

A ressonância magnética é considerada o método de imagem mais acurado para o diagnóstico de osteomielite ${ }^{17}$. Quando contraindicada ou indisponível, pode-se recorrer a métodos de medicina nuclear, sendo o principal a cintilografia óssea trifásica. Segundo metanálise de Dihn et al. ${ }^{19}$, a ressonância magnética e a cintilografia óssea trifásica possuem acurácia de $74 \%$ e $62 \%$, respectivamente, para o diagnóstico de osteomielite. A partir dos estudos discutidos no presente artigo, pode-se concluir que a cintilografia com ${ }^{99 m}$ Tc-ubiquicidina (29-41) é um método que possui elevada acurácia para o diagnóstico de osteomielite, com muitos estudos chegando a $100 \%$.

Os estudos de Emailiejah et al. ${ }^{9}$ e Beiki et al. ${ }^{10}$ revelaram que a cintilografia com ${ }^{99 m}$ Tc-ubiquicidina (2941) pode ser utilizada para o diagnóstico de infecções em implantes e próteses articulares e, segundo Ahktar et al. ${ }^{2}$, Gandomkar et al. ${ }^{3}$, e de Murphy et al. ${ }^{4}$, também possui elevada sensibilidade e especificidade no diagnóstico de infecção de tecidos moles. No entanto, os dados de Beiki et al. ${ }^{10}$ possuem uma discordância que gera dúvidas quanto a real acurácia do seu estudo. Os autores consideraram nula a quantidade de falsos-positivos, mas os valores descritos no trabalho geram uma interpretação diferente. Segundo exposto, a cintilografia ${ }^{99 \mathrm{~m}} \mathrm{Tc}$-ubiquidicina diagnosticou 6 pacientes com infecção em implante ortopédico, já a cultura confirmou apenas 5 desses pacientes como infectados, o que impossibilita obter-se o valor de especificidade relatado de $100 \%$, fato que alteraria consequentemente o valor preditivo positivo e a acurácia do estudo. Não foi possível concluir se os resultados relatados possuem algum equívoco ou se o estudo superestimou a acurária da cintilografia ${ }^{99 m}$ Tc-ubiquidicina, o que suscita dúvidas sobre a real confiabilidade dos resultados e conclusões obtidos a partir de sua análise.

Aryana et al. ${ }^{16}$ foi o único dos onze autores que comparou os resultados da análise das imagens pelos métodos qualitativo e semi-quantitativo. No seu estudo ambas as formas de análise apresentaram a mesma sensibilidade, porém a especificidade da análise semiquantitativa foi superior à da qualitativa. Essa conclusão é corroborada por um estudo de 2008 de Vallejo et al. ${ }^{20}$, sugerindo que a diferença de metodologia na análise dos estudos pode ter contribuído para a heterogeneidade das acurácias encontradas. Nos demais dez estudos, seis realizaram uma análise qualitativa e quatro uma análise semi-quantitativa.

Uma possibilidade para as diferenças de acurácia encontradas em diferentes estudos pode ser a variedade de agentes etiológicos das infecções. A infecção por Klebsiella e a infecção por E. coli podem diminuir a acurácia da cintilografia com ${ }^{99 \mathrm{~m}} \mathrm{Tc}$-ubiquicidina (29-41) segundo Emailiejah et al. ${ }^{9}$ e Akhtar et al. ${ }^{2}$, respectivamente. No entanto, essa análise é controversa, pois o estudo de Beiki et al. ${ }^{10}$ não encontrou relação entre o microrganismo e a acurácia do exame.

Estudos relataram que o uso de antibióticos previamente à realização do exame pode interferir na sua acurácia, pois a captação do radiofármaco pelo sítio infectado seria reduzida ${ }^{9-10}$. Além disso, parece haver um limiar quantitativo mínimo de concentração de bactérias para que o radiofármaco seja captado 9 . Fard-Esfahani et al. $^{5}$ sugerem que a baixa sensibilidade encontrada no estudo deles possa decorrer do fato da infecção dos seus pacientes serem crônicas. Dessa forma, a menor concentração tecidual de bactérias teria interferido na acurácia diagnóstica. Esses achados implicam que uma possível aplicação da cintilografia ${ }^{99 \mathrm{~m} T c-u b i q u i c i d i n a}$ seria o controle de cura em pacientes submetidos a 
antibioticoterapia?

Todos os estudos que avaliaram o momento mais adequado para a obtenção das imagens determinaram que imagens precoces possuem melhor acurácia do que imagens obtidas tardiamente. Um único estudo avaliou a obtenção das imagens em 15 minutos e concluiu que a acurácia é decrescente de 15 minutos até 240 minutos $^{14}$. Três estudos concluíram que o melhor momento seria aos 30 minutos $^{2,3,14}$, porém nenhum deles comparou com 15 minutos; outro estudo determinou que seria aos 60 minutos $^{9}$, porém ele não comparou com 15 ou 30 minutos. Dois estudos não encontraram diferença no tempo de obtenção das imagens para o diagnóstico ${ }^{5,10}$. Cabe ressaltar, no entanto, que esses dois estudos fizeram uma comparação apenas qualitativa dessa diferença, enquanto os demais estudos que encontraram diferença fizeram uma análise quantitativa. Dessa forma, um fator que pode ter contribuído para a heterogeneidade dos valores de especificidade do método cintilográfico pode ter sido o momento de aquisição das imagens.

\section{CONCLUSÃO}

A cintilografia com ${ }^{99 m}$ Tc-ubiquicidina (29-41) é um método de elevada acurácia para o diagnóstico diferencial entre infecções musculoesqueléticas e inflamações assépticas, sendo que 9 dos 11 estudos relataram acurácia $>90 \%$.

O curto intervalo entre a injeção do radiofármaco e obtenção das imagens (em geral 30 minutos) permite rápida definição diagnóstica e determinação de conduta médica, além de viabilizar sua aplicação na população pediátrica.

Esse método de cintilografia é uma técnica promissora com potencial de disseminação nos próximos anos, podendo se apresentar como uma alternativa viável à ressonância magnética quando ela estiver indisponível ou quando contraindicada.

Uma limitação do nosso estudo é que não foi realizada uma metanálise para determinar a sensibilidade e especificidade agrupada da cintilografia com ${ }^{99 \mathrm{~m}} \mathrm{Tc}-$ ubiquicidina (29-41).

Conflitos de interesse: Os autores declaram não possuir conflitos de interesse.

Participação dos autores: Silva, Carvalho, Alves e Azevedo contribuiram igualmente para a realização deste trabalho sob a orientação dos professores Campos, Netto e Bittencourt.

\section{REFERÊNCIAS}

1. Meléndez-Alafort L, Rodríguez-Cortés J, Ferro-Flores G, De Murphy CA, Herrera-Rodríguez R, Mitsoura E, et al. Biokinetics of 99mTc-UBI 29-41 in humans. Nucl Med Biol. 2004;31(3):373-9. doi: 10.1016/j.nucmedbio.2003.10.005.

2. Akhtar M, Qaisar A, Irfanullah J, Iqbal J, Khan B, Jehangir $\mathrm{M}$, et al. Antimicrobial peptide $99 \mathrm{mTc}$-Ubiquicidin $29-41$ as human infection-imaging agent: clinical trial. J Nucl Med. 2005;46:567-73. Available from: http://jnm.snmjournals.org/ content/46/4/567.long

3. Gandomkar M, Najafi R, Shafiei M, Mazidi M, Goudarzi $\mathrm{M}$, Mirfallah S, et al. Clinical evaluation of antimicrobial peptide [99mTc/Tricine/HYNIC0]ubiquicidin $29-41$ as a human-specific infection imaging agent. Nucl Med Biol. 2009;36(2):199-205. doi: 10.1016/j.nucmedbio.2008.11.003.

4. De Murphy CA, Gemmel F, Balter J. Clinical trial of specific imaging of infections. Nucl Med Commun. 2010;31(8):72633. doi: 10.1097/MNM.0b013e32833a3d7f.

5. Fard-Esfahani A, Beiki D, Fallani B, Mohajeri-Tehrani M, Gharaie M, Rouhipour N, et al. Evaluation of 99mTcUbiquicidin 29-41 scintigraphy in differentiation of bacterial infection from sterile inflammation in diabetic foot. Iran $\mathrm{J}$ Nucl Med. 2010;18(2):20-8. Available from: http://irjnm. tums.ac.ir/article_560_9e5594b9b2ed6edd34efc3ca5d9ea9 db.pdf.

6. Palestro C. Radionuclide imaging of osteomyelitis. Semin Nucl Med. 2015;45(1):32-46. doi: 10.1053/j. semnuclmed.2014.07.005.
7. Akhtar M, Imran M, Nadeem M, Shahid A. Antimicrobial peptides as infection imaging agents: better than radiolabeled antibiotics. Int J Pept. 2012;2012:1-19. doi: $10.1155 / 2012 / 965238$.

8. Hiemstra PS, Van den Barselaar MT, Roest M, Nibbering PG, van Furth R. Ubiquicidin, a novel murine microbicidal protein in the cytosolic fraction of the activated macrophages. J Leukocyte Biol.1999;66:423-8. Available from: https:// www.ncbi.nlm.nih.gov/pubmed/10496312.

9. Esmailiejah AA, Abbasian M, Azarsina S, Safdari F, Amoui M, Hosseinzadeh S. Diagnostic efficacy of UBI scan in musculoskeletal infections. Arch Iran Med. 2015;18(6):3715. doi: 015186/AIM.009.

10. Beiki D, Yousefi G, Fallahi B, Tahmasebi MN, Gholamrezanezhad A, Fard-Esfahani A, et al. ${ }^{99 m}$ TcUbiquicidin [29-41], a promising radiopharmaceutical to differentiate orthopedic implant infections from sterile inflammation. Iran J Pharm Res. 2013;12(2):347-53. Available from: https://www.ncbi.nlm.nih.gov/pmc/articles/ PMC3813225/pdf/ijpr-12-347.pdf.

11. Ocampo IZ. Comparação e validação de técnicas clássicas e modificadas para estudos de potencial genotóxico de peptídeos utilizados na produção de radiofármacos [dissertação]. São Paulo: Instituto de Pesquisas Energéticas e Nucleares, Universidade de São Paulo; 2016. Disponível em:http://www.teses.usp.br/teses/disponiveis/85/85131/ tde-18042016-103044/pt-br.php. 
12. Dias L. Desenvolvimento de um radiofármaco para marcação com Tc-99m para a identificação de infecção utilizando um peptídeo catiônico sintético [tese]. São Paulo: Instituto de Pesquisas Energéticas e Nucleares, Universidade de São Paulo; 2015. Disponível em: http://www.teses.usp.br/teses/ disponiveis/85/85131/tde-11012016-135639/en.php.

13. Saeed S, Zafar J, Khan B, Akhtar A, Qurieshi S, Fatima $\mathrm{S}$, et al. Utility of $99 \mathrm{mTc}$-labelled antimicrobial peptide ubiquicidin (29-41) in the diagnosis of diabetic foot infection. Eur J Nucl Med. 2013;40(5):737-43. doi: 10.1007/ s00259-012-2327-1.

14. Assadi M, Vahdat K, Nabipour I, Sehhat M, Hadavand F, Javadi H, et al. Diagnostic value of $99 \mathrm{mTc}$-ubiquicidin scintigraphy for osteomyelitis and comparisons with 99mTc-methylene diphosphonate scintigraphy and magnetic resonance imaging. Nucl Med Commun. 2011;32(8):71623. doi: 10.1097/MNM.0b013e3283483fe4.

15. Dillmann-Arroyo C, Cantú-Leal L, Campa-Núñez H, López-Cavazos C, Bermúdez-Argüelles M, Mejía-Herrera JC. Utilidad de la gammagrafía con ubiquicidina 29-41 en el diagnóstico de osteomielitis piógena de columna vertebral. Acta Ortop Mex. 2011; 25(1):27-31. Available from: http:// www.medigraphic.com/pdfs/ortope/or-2011/or111f.pdf.
16. Aryana K.; Hootkani A.; Sadeghi R.; Davoudi Y.; Naderinasab M.; Ayati N. ${ }^{99 \mathrm{~m} T c-}$ Labeled Ubiquicidin scintigraphy: A promising method in hip prosthesis infection diagnosis. Nuklearmedizin. 2012;51(4):133-9. doi: 10.3413/ Nukmed-0444-11-11.

17. Markanday A. Diagnosing diabetic foot osteomyelitis: narrative review and a suggested 2-Step Score-Based Diagnostic Pathway for Clinicians. Open Forum Infect Dis. 2014;1(2):1-6. doi: 10.1093/ofid/ofu060.

18. Bori G, Muñoz-Mahamud E, Garcia S, Mallofre C, Gallart X, Bosch J, et al. Interface membrane is the best sample for histological study to diagnose prosthetic joint infection. Mod Pathol. 2010;24(4):579-84. doi: 10.1038/ modpathol.2010.219.

19. Dinh M, Abad C, Safdar N. Diagnostic accuracy of the physical examination and imaging tests for osteomyelitis underlying diabetic foot ulcers: meta-analysis. Clin Infect Dis. 2008;47(4):519-27. doi: 10.1086/590011.

20. Vallejo E, Martinez I, Tejero A, Hernandez S, Jimenez L, Bialostozky D, et al. Clinical utility of $99 \mathrm{mTc}-$ Labeled Ubiquicidin 29-41 antimicrobial peptide for the scintigraphic detection of mediastinitis after cardiac surgery. Arch Med Res. 2008;39(8):768-74. doi: 10.1016/j. arcmed.2008.09.002 